\title{
Prophylactic Systemic Antifungal Agents to Prevent Mortality and Morbidity in Very Low Birth Weight Infants
}

\section{Cochrane Abstract}

Background: Invasive fungal infection is an important cause of mortality and morbidity in very low birth weight infants. Early diagnosis is difficult and treatment is often delayed. Systemic antifungal agents (usually azoles) are increasingly used as prophylaxis against invasive fungal infection. Objectives: To assess the effect of prophylactic systemic antifungal therapy on mortality and morbidity in very low birth weight infants. Search Methods: We used the standard search strategy of the Cochrane Neonatal Review Group. This included searches of the Cochrane Central Register of Controlled Trials (CENTRAL) (The Cochrane Library 2012, Issue 3), MEDLINE, EMBASE, and CINAHL (to August 2012), conference proceedings, and previous reviews. Selection Criteria: Randomized controlled trials or quasi-randomized controlled trials that compared the effect of prophylactic systemic antifungal therapy versus placebo or no drug or another antifungal agent or dose regimen in very low birth weight infants. Data Collection and Analysis: We extracted data using the standard methods of the Cochrane Neonatal Review Group, with separate evaluation of trial quality and data extraction by two review authors. Main Results: We identified 11 eligible trials enrolling a total of 1,136 infants. Seven trials (involving 880 infants) compared systemic antifungal prophylaxis versus placebo or no drug. These trials were generally small but of good methodological quality.
Meta-analysis found a statistically significant reduction in the incidence of invasive fungal infection in infants who received systemic antifungal prophylaxis (typical risk ratio (RR) $0.41,95 \%$ confidence interval $(\mathrm{Cl}) 0.27-0.61$; risk difference (RD) $-0.09,95 \% \mathrm{Cl}-0.14$ to -0.05$)$. The average incidence of invasive fungal infection in the control groups of the trials (16\%) was much higher than that generally reported from large cohort studies $(<5 \%)$. Meta-analysis did not find a statistically significant difference in the risk of death prior to hospital discharge (typical RR $0.74,95 \% \mathrm{Cl} 0.52-1.05$; RD $-0.04,95 \% \mathrm{Cl}-0.08$ to 0.01 ). Very limited data on long-term neurodevelopmental outcomes were available. Two trials that compared systemic versus oral or topical non-absorbed antifungal prophylaxis did not detect any statistically significant effects on invasive fungal infection or mortality. Two trials that compared different dose regimens of prophylactic intravenous fluconazole did not detect any significant differences in infection rates or mortality.

\section{Reviewers' Conclusions}

Prophylactic systemic antifungal therapy reduces the incidence of invasive fungal infection in very low birth weight infants. This finding should be interpreted and applied cautiously since the incidence of invasive fungal infection was

\section{KARGER}

E-Mail karger@karger.com

www.karger.com/neo
(C) 2013 S. Karger AG, Basel

1661-7800/13/1052-0095\$38.00/0 
very high in the control groups of most of the included trials. Meta-analysis does not demonstrate a statistically significant effect on mortality. There are currently only limited data on the long-term neurodevelopmental consequences for infants exposed to this intervention. In addition, there is a need for further data on the effect of the intervention on the emergence of organisms with antifungal resistance.

Austin N, McGuire W: Prophylactic systemic antifungal agents to prevent mortality and morbidity in very low birth weight infants. Cochrane Database of Systematic Reviews 2013, Issue 4. Art. No.: CD003850. DOI: 10.1002/14651858.CD003850.pub4.

\section{Commentary}

Roger F. Soll, Burlington, Vt.

Just because something works does not mean you have to use it. In their updated Cochrane Review, Austin and McGuire [1] identified seven trials (involving 880 infants) that compared systemic antifungal prophylaxis to placebo or no drug. The meta-analysis found no statistically significant decrease in the risk of death (fig. 1) but did report a statistically significant reduction in the risk of invasive fungal infection in infants who received systemic antifungal prophylaxis (typical risk ratio $0.41,95 \%$ CI $0.27-0.61$; risk difference $-0.09,95 \%$ CI -0.14 to -0.05 ) (fig. 2). However, the clinical significance of this apparently impressive result remains uncertain. As the authors note, the average incidence of invasive fungal infection in the control group in these trials was much higher than those generally reported in large cohort studies. In the Vermont Oxford Network, the mean rate of fungal infection in 2011 for extremely low birth weight infants enrolled in the expanded database was $2.1 \%$ (first quartile $0 \%$, third quartile of $2.1 \%$ ) [2]. The rate of infection in the control group makes these study centers extreme outliers regarding the incidence of fungal infection.

Antifungal prophylaxis is still recommended in neonatal units with high rates of invasive fungal infection [3, 4]. The American Academy of Pediatrics Red Book recommends fluconazole prophylaxis in extremely low birth weight infants cared for in neonatal intensive care units with moderate $(5-10 \%)$ or high $(>10 \%)$ rates of invasive candidiasis [3]. As noted above, few centers will have such extreme rates of infection. Importantly, the Red Book also notes that inadequate infection prevention practices and injudicious use of antimicrobial agents may contribute to high rates of fungal sepsis. Adherence to optimal infec- tion control practices, including appropriate practices for intravascular catheter insertion and maintenance, and antimicrobial stewardship could diminish the rates of infection and should be optimized before implementation of chemoprophylaxis as standard practice in the neonatal intensive care unit. This strikes at the heart of the matter. The extraordinarily high rates of fungal infection in the control group of the studies as well as those suggested as cut-off points for use of fluconazole prophylaxis support the argument that careful infection control procedures are a better way of combating invasive fungal infections in extremely preterm infants. The neonatal community must commit to the most basic of appropriate care in order to minimize these risks, not resort to expensive and potentially harmful drug therapy.

\section{Acknowledgment}

Editorial support of the Cochrane Neonatal Review Group has been funded with Federal Funds from the Eunice Kennedy Shriver National Institute of Child Health and Human Development, National Institutes of Health, Department of Health, USA, under Contract No. HHSN275201100016C.

\section{References}

1 Austin N, McGuire W: Prophylactic systemic antifungal agents to prevent mortality and morbidity in very low birth weight infants. Cochrane Database Syst Rev 2013, Issue 4. Art. No.: CD003850. DOI: 0.1002/ 14651858.CD003850.pub4.

2 Database of all infants admitted to neonatal intensive care units. Burlington, Vermont Oxford Network, 2013. https://nightingale.vtoxford.org (accessed on May 28, 2013).

3 American Academy of Pediatrics Red Book 2012; Pickering LK (ed): Report of the Committee on Infectious Diseases, ed 29. Elk Grove Village, AAP, 2012.

-4 Sweet DG, Carnielli V, Greisen G, Hallman M, Q-Ozek E, Plavka R, Saugstad OD, Simeoni U, Speer CP, Vento M, Halliday HL: European consensus guidelines on the management of neonatal respiratory distress syndrome in preterm infants - 2013 update. Neonatology 2013;103:353368.
Cochrane Review Update 


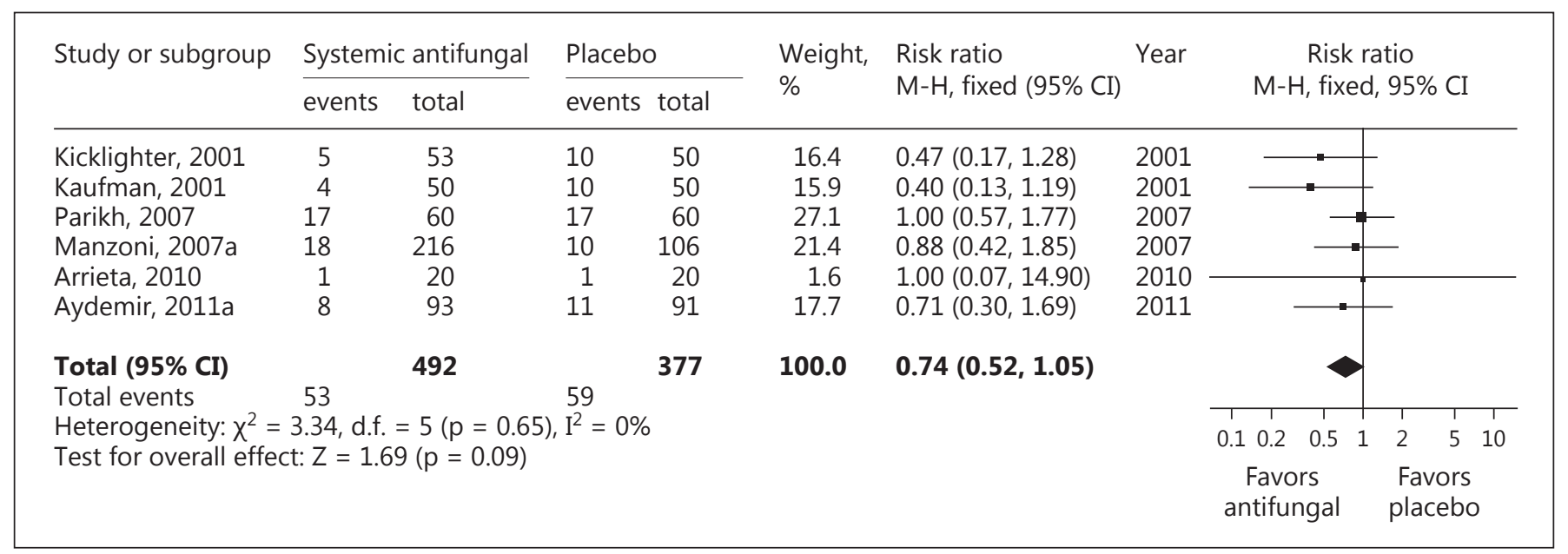

Fig. 1. Prophylactic systemic antifungal agents to prevent mortality and morbidity in very low birth weight infants. Effect on death prior to hospital discharge.

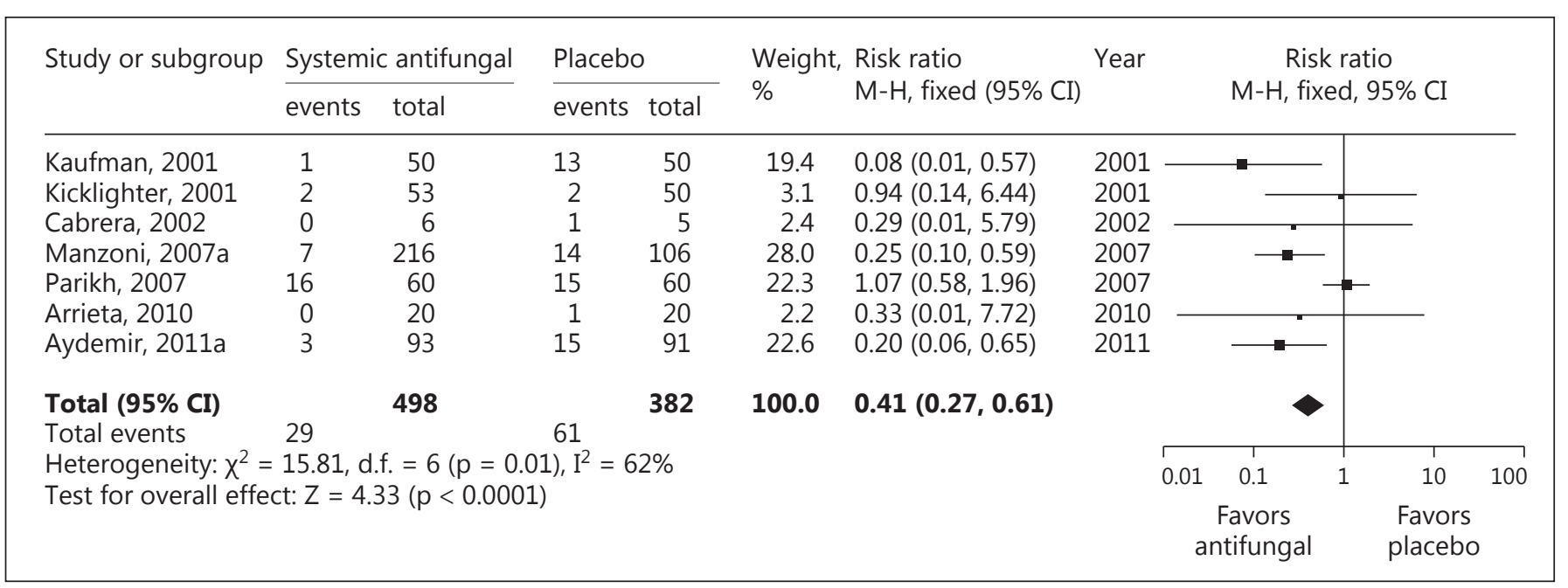

Fig. 2. Prophylactic systemic antifungal agents to prevent mortality and morbidity in very low birth weight infants. Effect on invasive fungal infection. 